\title{
Optimization of power production and costs in microgrids
}

\section{R. De Leone, A. Giovannelli \& M. Pietrini}

\section{Optimization Letters}

ISSN 1862-4472

Optim Lett

DOI 10.1007/s11590-016-1010-z

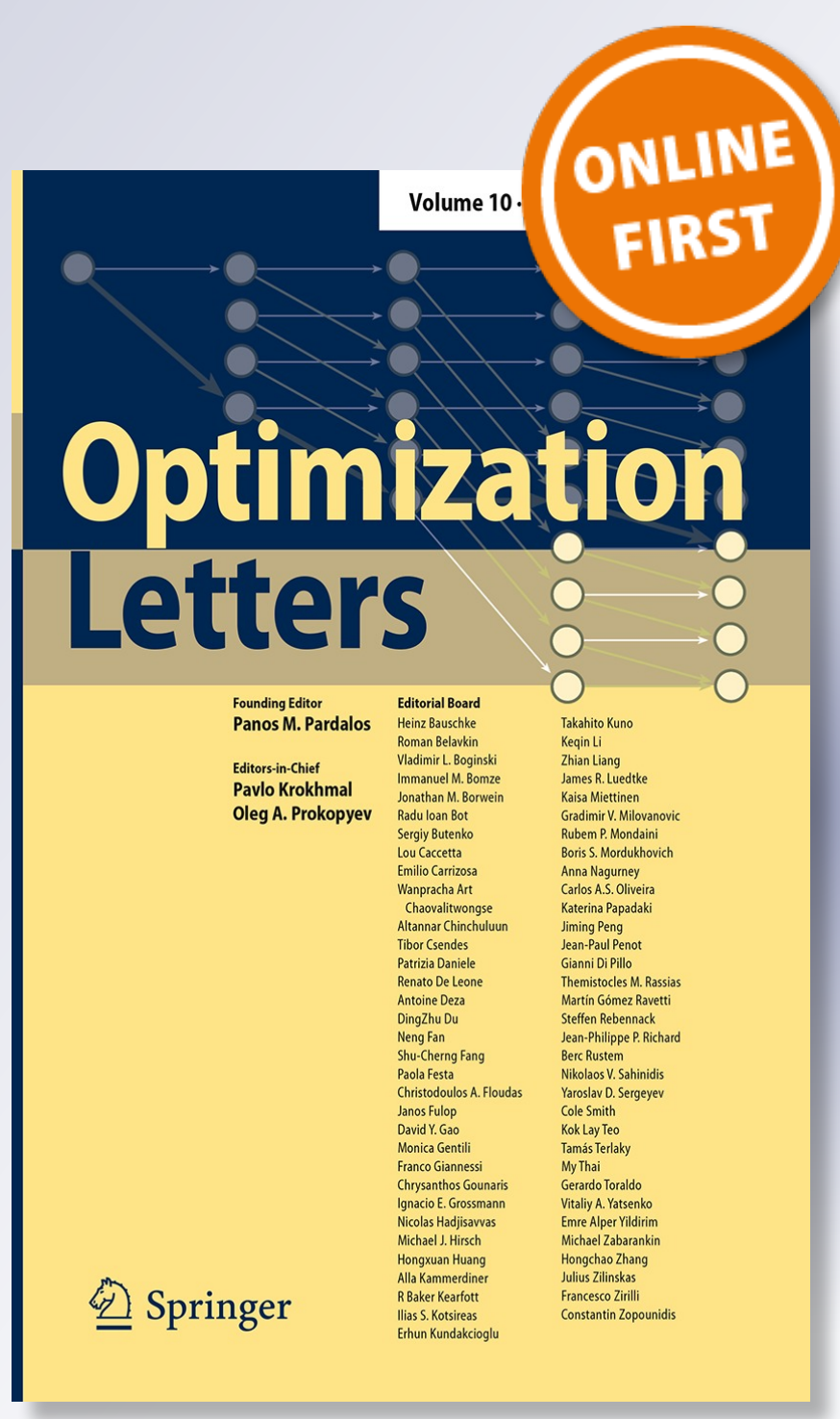

照 Springer 
Your article is protected by copyright and all rights are held exclusively by SpringerVerlag Berlin Heidelberg. This e-offprint is for personal use only and shall not be selfarchived in electronic repositories. If you wish to self-archive your article, please use the accepted manuscript version for posting on your own website. You may further deposit the accepted manuscript version in any repository, provided it is only made publicly available 12 months after official publication or later and provided acknowledgement is given to the original source of publication and a link is inserted to the published article on Springer's website. The link must be accompanied by the following text: "The final publication is available at link.springer.com". 


\title{
Optimization of power production and costs in microgrids
}

\author{
R. De Leone ${ }^{1} \cdot$ A. Giovannelli ${ }^{2} \cdot$ M. Pietrini ${ }^{1}$
}

Received: 21 May 2015 / Accepted: 19 January 2016

(C) Springer-Verlag Berlin Heidelberg 2016

\begin{abstract}
This paper proposes a mathematical formulation for energy management in a connected microgrid. The aim is determining the optimal operating strategy for energy storage, to fulfil a time-varying energy demand and operational constraints while achieving a tradeoff between microgrid running costs and energy storage system life. The microgrid is composed by various renewable power production plants, storage devices and controllable loads, and has the ability to increase energy efficiency and reduce costs for energy purchasing from the main grid. The problem is formulated as a mixed-integer linear optimization problem. The optimization is aimed at minimizing the overall cost function of the system while satisfying the customer demand and safety of the electrical network. A case study of an existing microgrid is investigated: the microgrid consists of a photovoltaic and a hydroelectric power plant, a battery storage, an office building and an industrial facility. The optimization problem is solved in an efficient way by using commercial software. Simulation results show the feasibility and the effectiveness of the proposed approach to satisfy the load and reduce total costs.
\end{abstract}

Keywords Electrical microgrid · MILP optimization · Energy management

$凶 \quad$ R. De Leone

renato.deleone@unicam.it

A. Giovannelli

a.giovannelli@loccioni.com

M. Pietrini

maila.pietrini@studenti.unicam.it

1 Mathematics Division, School of Science and Technologies, University of Camerino, Camerino, MC, Italy

2 Research@Energy, Loccioni Group, Via Fiume 16, Angeli di Rosora, AN, Italy 
Abbreviations

Problem data

$\alpha(t) \quad$ Energy purchased price at time $t(€ / \mathrm{kWh})$

$c I N D(t) \quad$ Energy consumption of the industrial facility at time $t(\mathrm{kWh})$

$c O F(t) \quad$ Energy consumption of the office building at time $t(\mathrm{kWh})$

$e(0) \quad$ Energy present in the battery storage at time $0(\mathrm{kWh})$

$p P V(t) \quad$ Photovoltaic energy production at time $t(\mathrm{kWh})$

$p H D(t) \quad$ Hydropower production at time $t(\mathrm{kWh})$

$v(t) \quad$ Energy selling price at time $t(€ / \mathrm{kWh})$

\section{Problem variables}

$\begin{array}{ll}b_{1}(t) & \text { Energy to buy for Block } 1 \text { at time } t(\mathrm{kWh}) \\ b_{2}(t) & \text { Energy to buy for Block } 2 \text { at time } t(\mathrm{kWh}) \\ e(t) & \text { Amount of energy present in the battery storage at time } t(\mathrm{kWh}) \\ \Theta_{b}(t) & \text { Buying/not buying energy at time } t(\mathrm{kWh}) \\ \Theta_{s}(t) & \text { Selling/not selling energy at time } t(\mathrm{kWh}) \\ r_{12}(t) & \text { Residual energy from Block } 1 \text { to Block } 2 \text { at time } t(\mathrm{kWh}) \\ s(t) & \text { Energy to sell at time } t(\mathrm{kWh}) \\ x_{1}(t) & \text { Energy sent to the battery storage from Block } 1 \text { at time } t(\mathrm{kWh}) \\ x_{2}(t) & \text { Energy sent to the battery storage from the main grid at time } t(\mathrm{kWh}) \\ y_{1}(t) & \text { Energy to discharge from the battery storage to Block } 1 \text { at time } t(\mathrm{kWh}) \\ y_{2}(t) & \text { Energy to discharge from the battery storage to Block } 2 \text { at time } t(\mathrm{kWh}) \\ \Omega_{C}(t) & \text { Charging state of the battery storage at time } t \\ \Omega_{D}(t) & \text { Discharging state of the battery storage at time } t\end{array}$

\section{Problem parameters}

$c I N V \quad$ Energy consumption of the inverter (kWh)

$m \quad$ Minimum energy to charge/discharge the battery storage (kWh)

$M \quad$ Maximum energy to charge/discharge the battery storage (kWh)

$m_{E S S} \quad$ Minimum energy capacity of the battery storage (kWh)

$M_{E S S} \quad$ Maximum energy capacity of the battery storage (kWh)

$\eta_{C} \quad$ Charging efficiency of the battery storage

$\eta_{D} \quad$ Discharging efficiency of the battery storage

$\eta_{T 1} \quad$ Efficiency of the transformer T1

$\eta_{T 2} \quad$ Efficiency of the transformer T2

$\hat{M} \quad$ Modeling constant 


\section{Introduction}

Energy has always been a key element in human life. Until now, its production has been entrusted with fossil fuels, but the ever-increasing energy demand has pointed out the limit of these resources. Consequence of this is the development, especially in recent decades, of plants producing energy from renewable sources: solar, thermal, and hydroelectric. Moreover, increasing attention has shifted to the new concepts of energy efficiency, rationalization of resources and environmental awareness.

In this context, distributed energy generation [1], especially solar and wind power collected in different small generation locations, has gained considerable importance and the use of renewable energy sources is perceived as vital in achieving carbon reduction goals $[15,18]$. As a consequence, nowadays the development of electrical microgrids is widely spreading. A microgrid (MG) is an electrical distribution network comprising various distributed power generators, storage devices and controllable loads that can operate either in a interconnected way or isolated from the utility service provider as a controlled entity. The microgrid is aimed to manage energy flows in order to efficiently distribute electricity and to obtain a more rational use of it minimizing, at the same time, incidental overloads and costs for the purchase of energy from the main power grid. The overall goal of an electrical microgrid is to achieve autonomy, thanks to its power generation facilities that must meet the loads required by the several buildings connected on the network. This paper assumes that the microgrid could operate connected to the uppergrid: it can purchase power from utility providers when the production of the MG is insufficient to meet the load demand and it can sell power to the main grid if the production is higher than consumption. There is a daily income to the MG when the generated power exceeds the load demand; moreover, it is assumed that it could draw unlimited power from the utility grid. An excess of energy produced by renewable sources can be either sold to the grid, or used to charge batteries, or a combination of the two.

The scientific challenge is to solve the environmental and economic problem in the power generation and in [12] an overview is reported of the commonly used algorithms that have been effectively solved by multiobjective evolutionary [2] and fuzzy satisfaction-maximizing approach [8]. Several strategies have been reported in the literature, related to the operation costs as well as minimizing emissions of microgrids. In [3] and [11] the problem is treated as a single objective problem and the emission, operation and maintenance costs are not taken into account as well as the issue of selling or purchasing power to or from the main distribution grid. Other microgrid scheduling problems have been solved through mixed integer linear optimization as in $[10,14,16,17]$.

A real-world case study is presented in this paper. The goal is to minimize the cost of the energy flows in an existing microgrid, called Leaf Community ${ }^{1}$, consisting of a photovoltaic and a hydroelectric power plant, a battery storage, an office building and an industrial facility (see Fig. 1 for a schematic representation). The management of the MG units requires an accurate economic model to describe the operating cost taking

\footnotetext{
1 Available at Loccioni Group, Angeli di Rosora, AN, Italy.
} 
into account the output energy produced. Such a model is discrete and nonlinear in nature, hence optimization tools are needed to reduce the operating costs to a minimum level [13]. The problem is formulated as a mixed-integer linear optimization problem, which is solved in an efficient way by using commercial solvers (MPL software with CPLEX solver). The optimization is aimed at minimizing the total cost of the system while satisfying the customer demand and safety of the system. Moreover, the battery allows to store energy when its cost is low and release it when the cost is high, but it is very crucial not to overuse it because any charge/discharge cycle would lead to energy losses. The overall idea is, by knowing the meteorological forecasts for the next 24 or $48 \mathrm{~h}$, to dispatch the energy in the microgrid in order to be able to satisfy the scheduled hourly consumptions and achieve a proper management of the energy storage. The study considers two different energy storage strategies: a-priori optimal strategy, in which the energy charging/discharging range of the storage system is established before solving the problem, and a-posteriori optimal strategy, where this range is not firstly established.

The paper is organized in the following way: the next section shows the microgrid topology and an analysis of the system model, then the preprocessing data introduces the formulation of the problem, explained with different strategy solutions. Finally, Sect. 5 reports simulation results showing good performance, then conclusions are pointed out.

\section{System model}

Microgrids offer an alternative to utility planners in their search for the best economically solution to electric supply problems [13]. In our study, we consider the MG system schematically represented in Fig. 1, called Leaf Community. The Leaf Community is a microgrid realized by Loccioni Group (Angeli di Rosora, An, Italy) and it consists of energy production systems from renewable sources (a photovoltaic plant

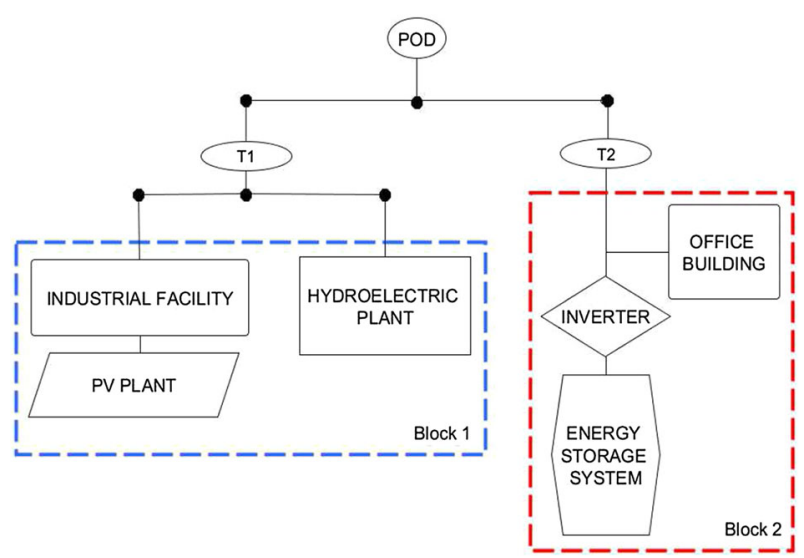

Fig. 1 The Leaf Community microgrid architecture scheme 

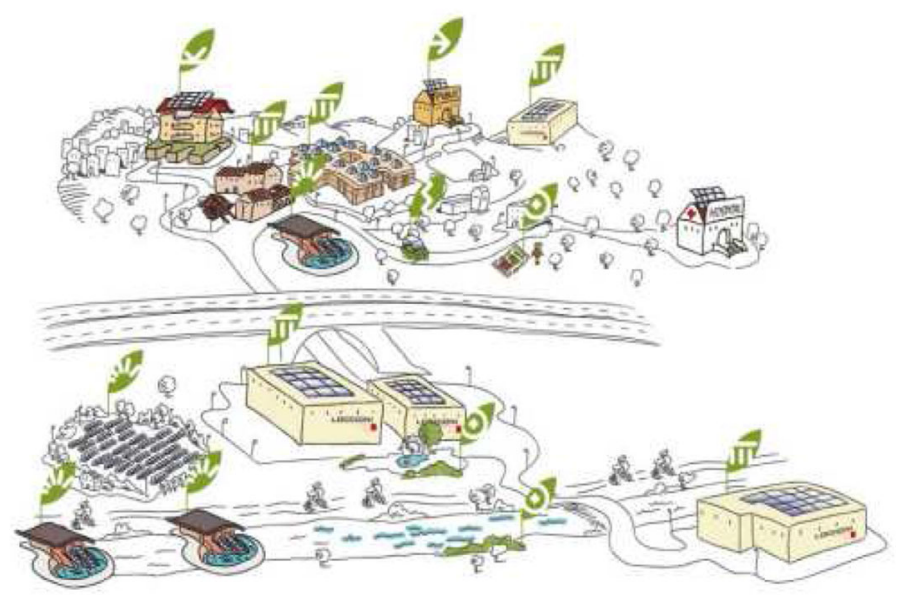

Fig. 2 The Leaf Community microgrid by Loccioni Group. Obviously, not all the plants are connected to the grid, yet

and a hydroelectric power station), consumption buildings (an industrial facility and an office building) and an energy storage device (ESS).

The photovoltaic plant has a total area of 1600 square meters with a nominal power of $148 \mathrm{kWp}$. The plant is installed on the roof of the industrial building and consists of cylindrical panels, which have the advantage of not being too affected by the temperature of the panel itself in the production of energy. The micro-hydroelectric plant produces an average of about $50 \mathrm{~kW}$ of power per day from October to May, while in summer the production is drastically reduced, because the power plant is positioned along the river Esino and the energy produced strongly depends on the water flow of the river throughout the year. The two consumption buildings of the MG are respectively an industrial plant with a surface area of 4000 square meters and a smaller building with only four rooms inside. The ESS is a lithium battery with a capacity of $224 \mathrm{~kW}$ of power controlled by an inverter able to ramp from full power charge to full power discharge in few seconds. There is a single point of connection to the utility main grid, called POD (Point of Delivery).

The single components of the MG are organized in two blocks: a first block composed by the industrial building, the PV and the hydroelectric plant; a second block composed by the energy storage system with its inverter and the office building. These two blocks are connected to the POD by two transformers (T1 and T2) of different dimensions in terms of apparent power of an AC system, 1250 and $630 \mathrm{kVA}$ respectively, that allow to switch from medium to low voltage: in fact, microgrids are Low Voltage distribution networks. Figure 2 gives a more realistic idea on the Leaf Community Microgrid. 


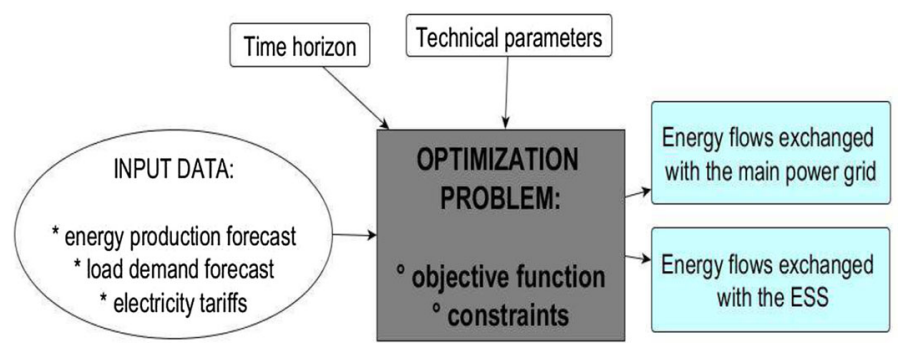

Fig. 3 The flowchart of the microgrid optimization problem

\section{Data preprocessing}

The microgrid optimization model will manage the energy flows within the network in order to ensure the maximum economic benefit and to achieve for most of the time the goal of energy autonomy for the overall system. The energy flows present in the microgrid are divided into

- energy production from renewable sources (solar, hydro);

- energy load demand by industrial and office buildings;

- energy to sell and to buy from the national grid;

- energy to be stored or to be taken from the storage system.

An overview of the optimization algorithm is shown in Fig. 3. The optimization problem takes as input the predicted values for production and consumption of the different buildings for the next one or two days ahead, the hourly purchased and selling electricity rates and some technical parameters, such as the roundtrip battery efficiency (power will be lost during the charging and discharging) and the dimensions of the two transformers. The output is the optimal quantity of energy to buy and sell to the national grid and the curves of energy to load/unload from the storage battery. Obviously, in order to maximize the gains from an economic point of view and amortize the total cost of the entire system battery-inverter, this problem must satisfy some constraints that will be discussed in detail in Sect. 4 .

As explained before, the global idea of the microgrid optimization problem is to manage the produced energy flows in order to ensure maximum auto-consumption taking into account the electricity rates and the possibility of using the storage battery. All this is on the basis of the forecasts of production from renewable sources and consumption expected one or two days ahead.

\subsection{The forecasting procedure}

Due to the stochastic nature of the weather and load demand, the predictions of production and consumption of the several plants has been obtained using standard forecasting techniques and Support Vector Machines.

Support vector regression is a well-proved machine learning approach for time series prediction $[5,6,20]$. It is based on statistical learning theory which generalizes 


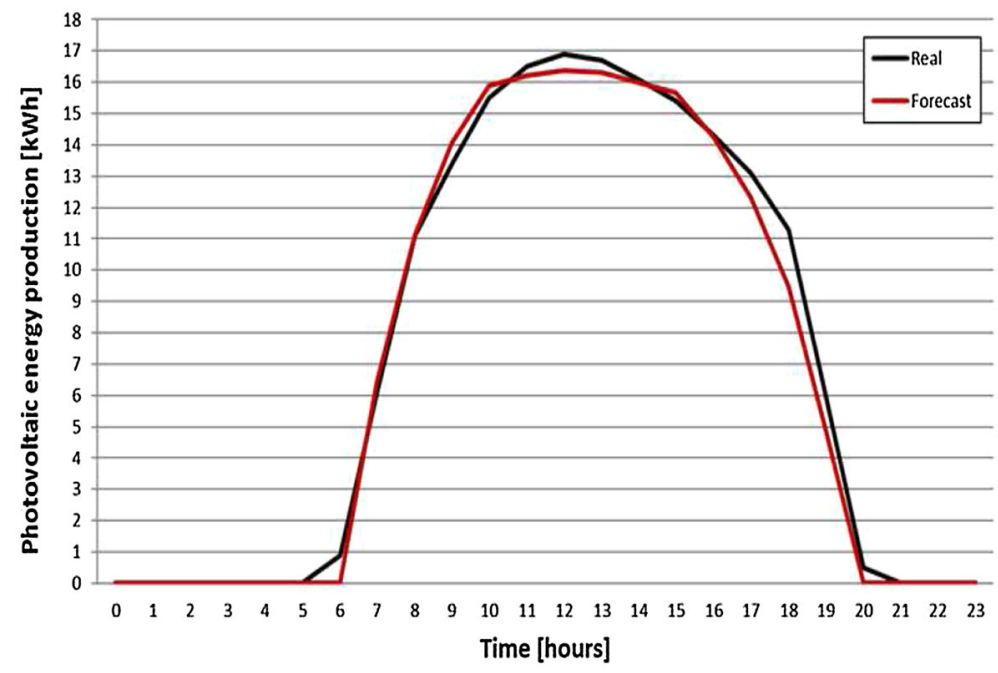

Fig. 4 The photovoltaic energy production forecast using SVM

well to unseen data. This technique has been used to forecast the energy production from the photovoltaic plant, according to the effect of the weather forecasts of the solar irradiance and the temperature for the next 24-48 $\mathrm{h}$ [7]. The model considers the external temperature and the solar irradiance as features in the training input data. Furthermore, there are some parameters to choose, which would influence the performance of the $v$-SVR algorithm: the cost of error, the kernel type and the number of support vectors $v$. In our experiments, since we assume a daily prediction and since the algorithm will be retrained every night in order to obtain the energy production forecast for the next day/s, we simply include the recorded irradiance, the temperature and the production data every 15 min of the previous fourteen days as training set. The only inputs the algorithm needs to forecast are the estimated irradiance and environmental temperature for the next $24 / 48 \mathrm{~h}$, provided by internet weather site. Obviously, the model is affected by the prediction error of the weather forecasts, because irradiance is the physical quantity mostly influencing the photovoltaic energy production. Figure 4 plots the real and forecasted photovoltaic energy production curves for a sunny day obtained with the $v$-SVR model.

Moreover, classical statistical techniques are used for energy production from hydroelectric power plant. In this case, an autoregressive model [19] was the best solution to forecast the produced energy for the next day: the change in the energy production varies about $1 \%$ from one day to another.

Finally, experiments showed that for the load demand of the industrial facility and the office building, statistical models can be also used. The Holt-Winters double exponential smoothing method [9] well estimated the cyclical nature of the consumptions during a typical week. Figure 5 shows the load demand forecast of the industrial building in a typical working day.

All these forecasted data are the inputs of the microgrid optimization algorithm, as reported in Fig. 3. 


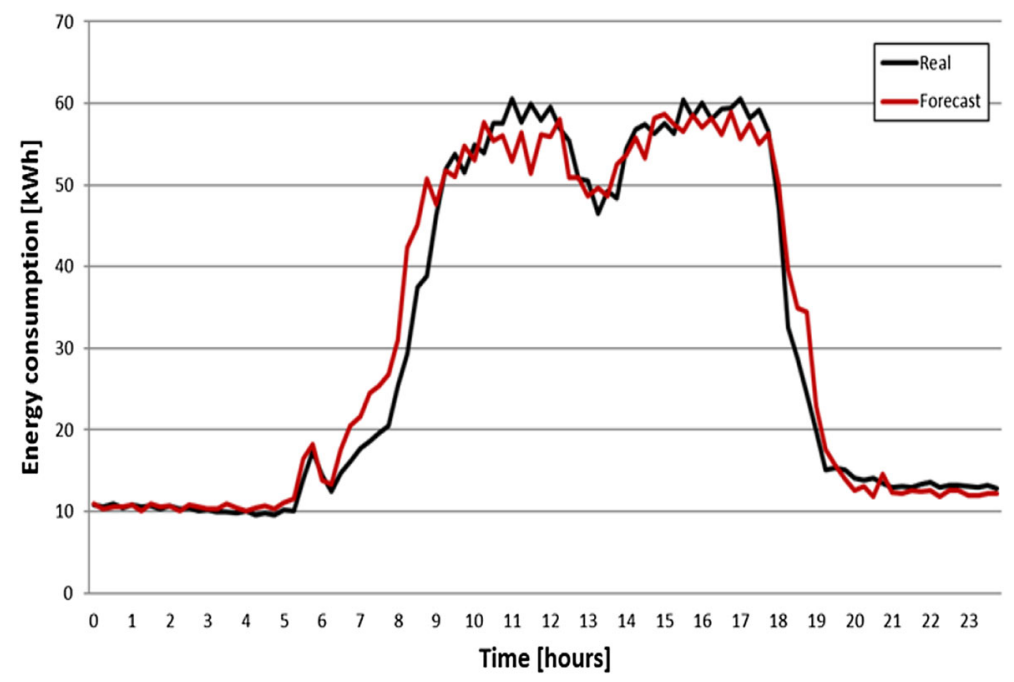

Fig. 5 The energy load demand forecast using Holt-Winters method

\subsection{Additional input data}

In addition to energy production and consumptions forecasts, the microgrid optimization algorithm takes as inputs other values. The management of the energy flows to be stored in the storage device or to be swapped with the national utility grid is highly dependent on the purchased electricity fare and rate of sale of energy in a day. The selling energy price can be considered constant (over the hours and days), while the purchased cost varies depending on the hour of day and changes from one day to another. The purchased price is normally disclosed at 12:00 of the previous day for the whole next day. Figure 6 shows the typical shape of the curve of prices during the day.

Moreover, other technical parameters are the following:

- the efficiency of the two transformers, related to their dimension and the mean power level they exchange;

- the inverter consumption, dependent on the use of the battery;

- the charging and discharging efficiency of the ESS;

- the initial state of charge of the battery and the swing range of the ESS to be referenced.

Table 1 shows the different possible scenarios for the management of the battery: such scenarios depend on the swing range of a cycle of charge and discharge of the battery and on the life of the energy storage system itself. The management framework of the battery is included in the technical sheet provided by the manufacturer. In Sect. 6 we will discuss two different strategies for the management of the battery: in the first case the swing target range is set a priori, while in the second case the procedure is to optimize the charge and discharge of the ESS. 


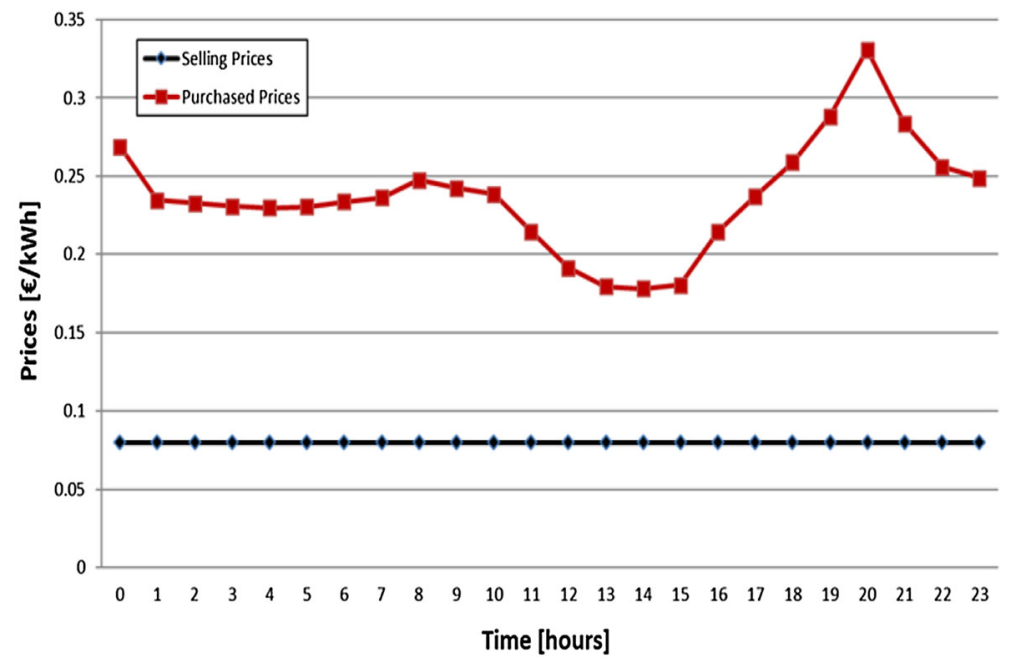

Fig. 6 The selling and purchased energy tariffs

Table 1 Management framework of the possible scenarios of the battery: in the first column the number of the possible scenarios is shown; the ranges (in \%) of the state of the lithium battery charge and discharge are pointed out in the second and third column; finally, the ESS life in terms of cycles is indicated in the last column

\begin{tabular}{lllr}
\hline No. scenario & SOC swing $\%$ & Swing range $(\%)$ & Cycle life \\
\hline 1 & 100 & $0-100$ & 6000 \\
2 & 75 & $25-100$ & 8400 \\
3 & & $0-75$ & 12,700 \\
4 & 50 & $50-100$ & 11,600 \\
5 & & $0-50$ & 36,100 \\
6 & 25 & $75-100$ & 49,500 \\
7 & & $50-75$ & 30,400 \\
8 & & $25-50$ & 76,100 \\
9 & & $0-25$ & $1,39,000$ \\
\hline
\end{tabular}

\section{Microgrid optimization problem formulation}

Each component of the Leaf Community microgrid is modeled separately, based on its characteristics and constraints. This section shows the model for each part of the MG system and the overall optimization problem formulation. The Microgrid Optimization Problem is formulated as a mixed-integer linear programming problem. The objective function and all constraints are linear and the problem involves continuous and binary variables.

Firstly, we assume that time is slotted, i.e. $t \in\{0,1, \ldots, 192\}$. The algorithm considers a time span of two days (easily extendable to three or more days) with data every $15 \mathrm{~min}$, therefore there are 192 time steps in the two days. Moreover, the 
algorithm is modular: the future introduction of variables for a new building or a production plant does not alter the setting of the system.

\subsection{Objective function}

The aim of the MG optimization problem is to minimize energy costs or, equivalently, to maximize gains, which is the approach utilized in this study. Following the general scheme reported in Sect. 2, energy is bought or sold from the main utility grid. Therefore, a cost is paid for the purchased energy whenever the generated output power from the renewable energy sources is insufficient to cover the load demand. On the other hand, there is an income because of sold energy to the main distribution grid when the power generated is higher than the consumptions of the MG but the price of the sold energy is lower than the purchased power tariff. For this reason, according to Fig. 1, the objective function takes the following form:

$$
\max \sum_{t=1}^{192}\left[v(t) s(t) \eta_{T 1}-\alpha(t)\left[b_{1}(t)+b_{2}(t)\right]\right]
$$

where the first term represents the total gains due to the energy sold to the main utility grid, where $v(t)$ is the energy selling price and $s(t) \eta_{T 1}$ is the amount of energy to sell left over the losses due to the transformer T1, while the second term represents the total costs due to the purchased energy from the main utility grid, where $\alpha(t)$ is the energy purchased price and $b_{1}(t)+b_{2}(t)$ is the sum of the energy needed to buy for the Block 1 and the Block 2 of the microgrid.

\subsection{Constraints}

\subsubsection{Energy balance constraints}

To meet the energy balance of the overall system, covering the total load demand with the total energy generated, two constraints for each $t \in\{1, \ldots, 192\}$ are imposed for the two blocks of the microgrid (Fig. 1). The sum of the energy purchased from utility grid for the two blocks, and the total energy generated by the different sources in the MG must be balanced by the local demand, the energy loss in the transmission lines and the energy in the storage system. More precisely, the energy balance equations are:

Block 1:

$p P V(t)+p H D(t)+b_{1}(t) \eta_{T 1}+y_{1}(t) \eta_{T 2} \eta_{T 1}=c I N D(t)+s(t) \eta_{T 1}+x_{1}(t) \eta_{T 1} \eta_{T 2}+r_{12}(t)$

Block 2:

$$
b_{2}(t) \eta_{T 2}+y_{2}(t)+r_{12}(t) \eta_{T 1} \eta_{T 2}=c O F(t)+x_{2}(t) \eta_{T 2} .
$$

$\forall t=1, \ldots, 192$, 
where

$p P V(t)$ and $p H D(t)$ are the renewable energy sources productions from photovoltaic and hydroelectric power plant, respectively;

$b_{1}(t)$ and $b_{2}(t)$ are the energy bought from the main grid to satisfy consumptions of the Block 1 and 2 respectively;

$y_{1}(t)$ and $y_{2}(t)$ are the energy drawn from the battery storage, in order to satisfy Block 1 and 2 respectively;

$s(t)$ is the energy sold to the main grid;

$c I N D(t)$ and $c O F(t)$ are the consumptions of the industrial and the office building; $x_{1}(t)$ and $x_{2}(t)$ are the amount of energy charged into the storage, coming from the Block 1 and the national grid, respectively;

$r_{12}(t)$ is a residual amount of energy from Block 1 to Block 2.

These quantities are multiplied to the efficiencies of the transformers present in the microgrid, $\eta_{T 1}$ and $\eta_{T 2}$. Notice that, for instance, in Eq. $2 x_{1}(t)$ is multiplied to both $\eta_{T 1}$ and $\eta_{T 2}$ because the point of view is internal to Block 1 .

\subsubsection{Battery storage constraints}

A battery is a storage device which can reserve additional energy. Whenever the PV and HD energy productions are insufficient to serve the demand load, energy in the ESS is needed and the battery will be discharged. On the other hand, there will be energy stored in the storage device whenever the supply from the renewable energy sources exceeds the consumptions. Meanwhile the charge and discharge of the battery and the total amount of energy sold to the main utility grid are monitored.

When determining the state of charge for an energy storage device, several constraint equations must be satisfied at all times. First, the energy present in the battery storage $e(t)$ is constrained by the following simple inequality, where $m_{E S S}$ and $M_{E S S}$ are the minimum and maximum energy capacity of the battery, respectively:

$$
m_{E S S} \leq e(t) \leq M_{E S S} \quad \forall t=0, \ldots, 192 .
$$

Obviously the cycles of charge $\left(x_{1}(t)+x_{2}(t)\right)$ and discharge $\left(y_{1}(t)+y_{2}(t)\right)$ of the battery must not exceed the limits established in the swing range of reference chosen a priori. The following inequalities express the constraints on the minimum $m$ and maximum $M$ amount of energy to charge or discharge from the battery storage:

$$
\begin{array}{ll}
m \leq x_{1}(t)+x_{2}(t) \leq M & \forall t=1, \ldots, 192 \\
m \leq y_{1}(t)+y_{2}(t) \leq M & \forall t=1, \ldots, 192 .
\end{array}
$$

The energy level $e(t)$ in the battery at time $t$ depends on the energy level of the previous time step, on the energy losses due to inefficiencies in the event of battery charge/discharge $\eta_{C}, \eta_{D}$, on the losses due to the transformers in the microgrid $\eta_{T 1}$, $\eta_{T 2}$ and on the consumption of the inverter $c I N V$. The inverter, in fact, has a such behavior, because consumes a constant amount of energy by taking it from the battery and not from the network. The following constraints model the energy level in the battery: 


$$
\begin{aligned}
e(t)= & e(t-1)+\eta_{C}\left[x_{1}(t) \eta_{T 1} \eta_{T 2}+x_{2}(t) \eta_{T 2}\right]-\Omega_{C}(t) c I N V \\
& -\eta_{D}\left[y_{1}(t) \eta_{T 1} \eta_{T 2}+y_{2}(t)\right]-\Omega_{D}(t) c I N V .
\end{aligned}
$$

$\forall t=1, \ldots, 192$ with $e(0)$ as input parameter.

The last constraint needed for the battery imposes the impossibility to simultaneously charge and discharge the ESS at the same time step $t$. In the optimization problem such condition has been set using two binary variables $\left(\Omega_{C}(t)\right.$ for charge, $\Omega_{D}(t)$ for discharge), which take the value 1 if it is, respectively, loading/unloading the battery, 0 otherwise. These relationships between binary variables $\left(\Omega_{C}(t), \Omega_{D}(t)\right)$ and continuous variables $\left(x_{1}(t), x_{2}(t)\right.$ for charge, $y_{1}(t), y_{2}(t)$ for discharge) are expressed by the following constraints:

$$
\begin{aligned}
& m \Omega_{C}(t) \leq x_{1}(t)+x_{2}(t) \leq M \Omega_{C}(t) \\
& m \Omega_{D}(t) \leq y_{1}(t)+y_{2}(t) \leq M \Omega_{D}(t)
\end{aligned}
$$

where $m, M$ are the minimum and maximum amount of energy to charge or discharge from the battery storage, respectively. Obviously at this point, the sum of these variables must not exceed 1:

$$
\Omega_{C}(t)+\Omega_{D}(t) \leq 1 \quad \forall t=1, \ldots, 192 .
$$

\subsubsection{Main grid constraints}

The last set of constraints considered in the model are related to the energy exchanges with the national utility service provider. The Leaf Community microgrid operates connected to the uppergrid: it can purchase energy when the renewable energy production is insufficient to meet the load demand and it can sell energy to the main grid if the production is higher than the consumptions. Obviously, it is impossible to purchase and sell energy at the same time $t$ : this condition is again expressed introducing two binary variables $\left(\Theta_{b}\right.$ for energy to buy, $\Theta_{s}$ for energy to sell), which take the value 1 if it is, respectively, buying/selling of energy, 0 otherwise. This condition is expressed by the following constraints:

$$
\begin{gathered}
\left\{\begin{array}{l}
\Theta_{b}(t) \leq \hat{M}\left(b_{1}(t)+b_{2}(t)\right) \\
b_{1}(t)+b_{2}(t) \leq \hat{M} \Theta_{b}(t)
\end{array}\right. \\
\left\{\begin{array}{l}
\Theta_{s}(t) \leq \hat{M} s(t) \\
s(t) \leq \hat{M} \Theta_{s}(t)
\end{array}\right.
\end{gathered}
$$

where $\hat{M}$ is a constant useful for modeling. Obviously, the sum of these variables must not exceed the value 1 at time step $t$ :

$$
\Theta_{b}(t)+\Theta_{s}(t) \leq 1 \quad \forall t=1, \ldots, 192
$$


Contractual constraints with the energy national grid manager dictate that only energy produced from renewable sources $(p P V(t)+p H D(t))$ can be sold to the uppergrid $s(t)$, not the energy stored in the battery, so the following constraint must be imposed:

$$
s(t) \leq p P V(t)+p H D(t) \quad \forall t=1, \ldots, 192 .
$$

\section{Computational results}

The optimization problem described in the previous section allows to manage the flows of energy within the Leaf Community microgrid while minimizing total costs. The time horizon is two days: all the results reported here refer to two different situations in order to underline the differences between working time and weekends. In the first cases a working day plus a day off are considered, in the other cases two working days are taken into account.

In the first part of this section we will report the computational results corresponding to the situation of the working day and the day off: 2 days in winter with the microhydro power plant in operation and two summer days without the energy production resulting from the micro-hydro. The swing range of the battery is set a priori between 0 and $25 \%$. The problem is applied to a time-varying energy load demand, with a range between 10 and $70 \mathrm{kWh}$ with time steps of $15 \mathrm{~min}$ for a working day, as shown in Fig. 5. For the first situation two scenarios are shown: one in which the energy storage system is initially fully charged, and the other in which it is fully discharged.

Figures 7 and 8 show the different behaviour of the energy flows obtained by solving the Microgrid Optimization Problem with different inputs. First, you can note that in both cases the photovoltaic and hydroelectric energy is first used to meet the required loads, then the two situations differ. If the battery is initially fully charged, the load demand is served with the battery storage when the electricity tariff is high and in the end of the first day the battery is fully discharged. From this result we can see that the renewable energy production plants are not large enough to supply the load for the whole time. Instead, if the battery is fully discharged, it is charged during the hours when the electricity tariffs is low, that is in the central hours of the day. In both cases, during the second day, on Saturday, when the production is bigger than the consumptions, the algorithm considers most economically profitable to sell energy rather than storing it in the battery: this depends on the fact that during the span of the second day is always over-production and there is no need to buy energy from the energy provider.

The last case corresponding to the situation of a working day plus a day off is illustrated in Fig. 9. It refers to two summer days, when the micro-hydro power station is inactive. In summer, energy consumptions are higher than in winter season and even the photovoltaic energy production is expected to be higher. The curves plotted in Fig. 9 point out the strategy of the Microgrid Optimization Problem: during the first day, the battery storage, originally discharged, is charged when the electricity tariffs are low. When the load demand is high and the purchased price is expensive, the best choice in term of cost is to start to use the energy stored in the ESS between 20:00 and 21:00. The second day shows a very profitable situation: the energy production is 
R. De Leone et al.

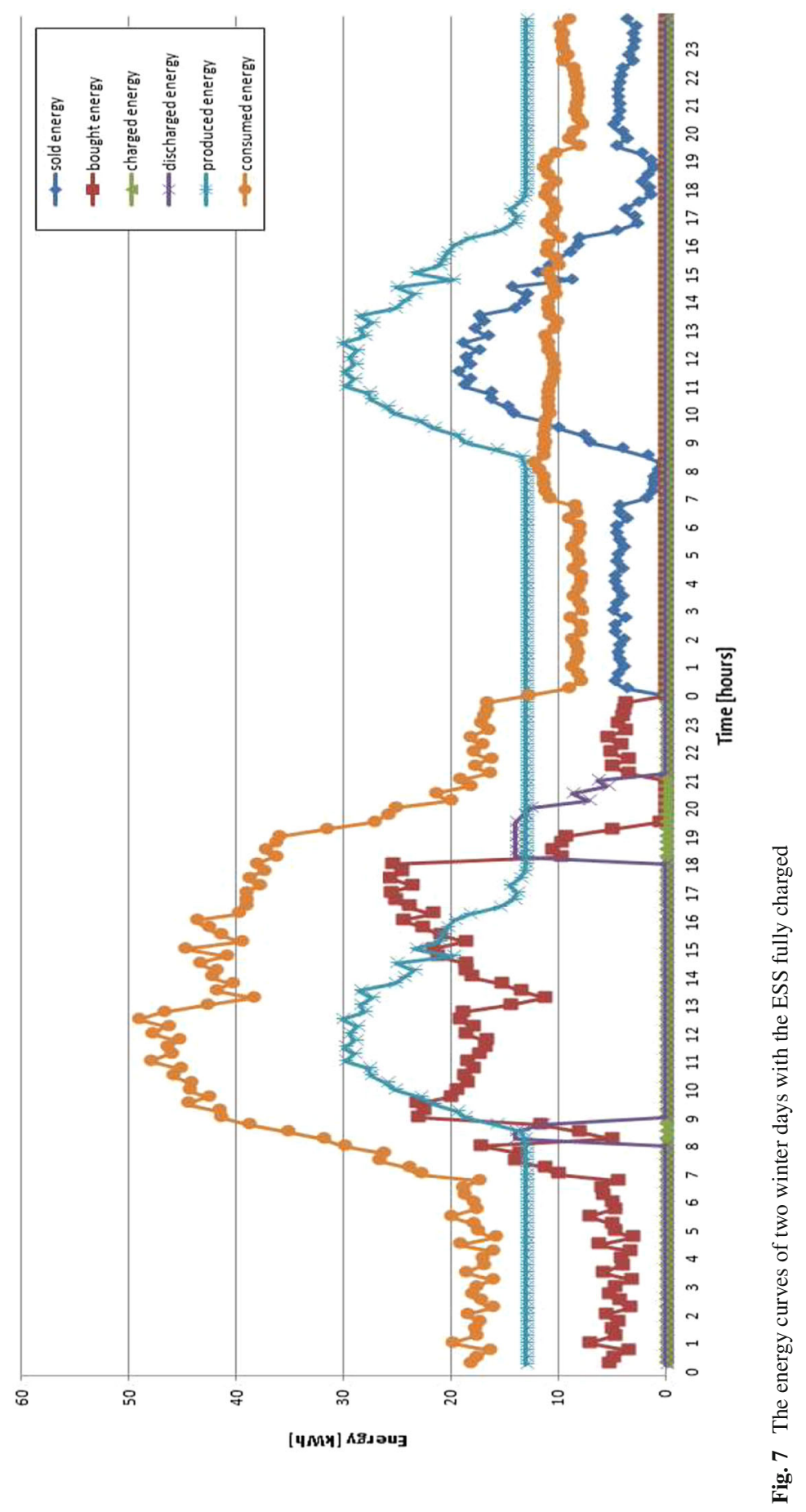




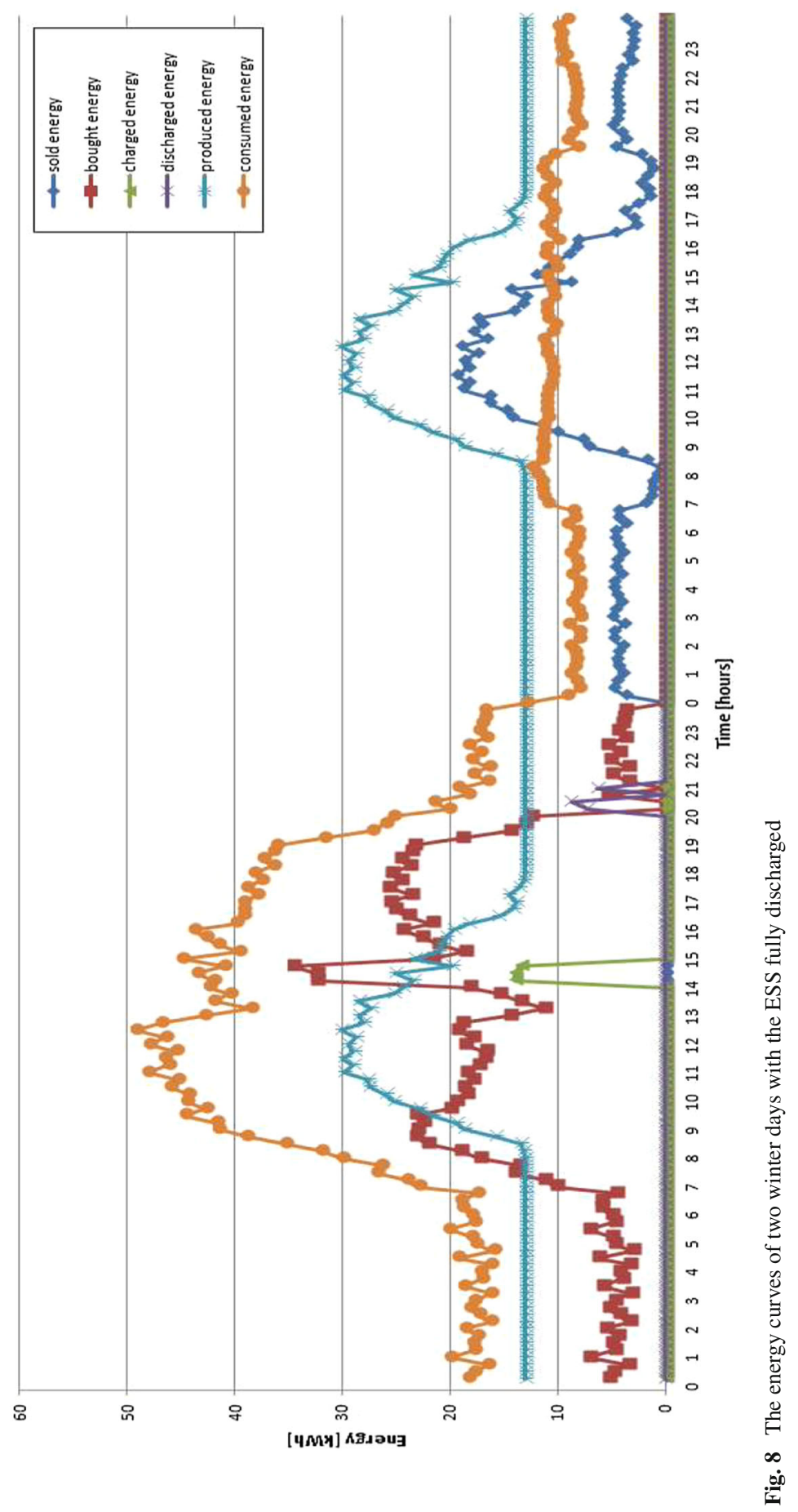


R. De Leone et al.

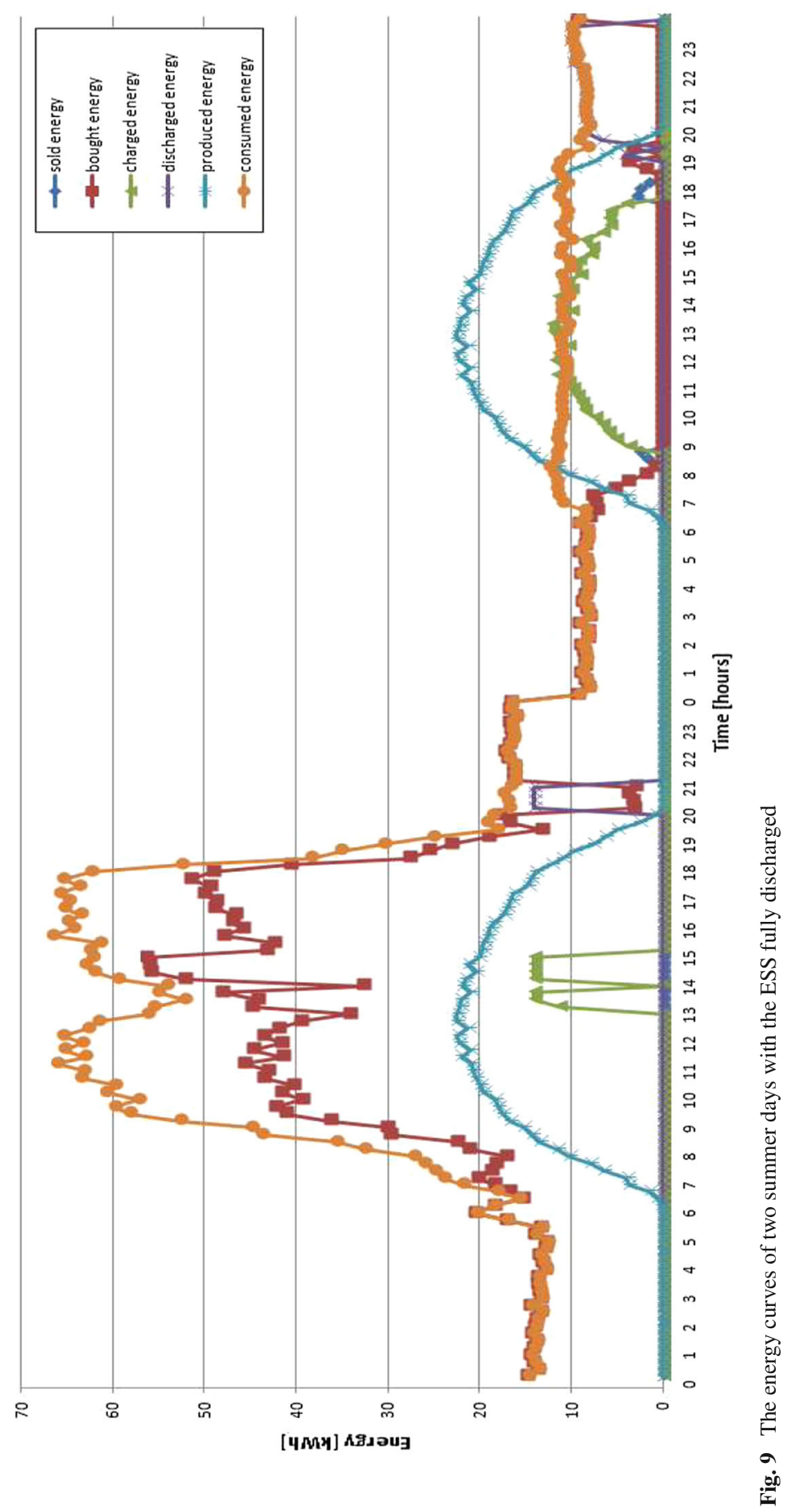


bigger than consumptions and the battery can be charged during the daylight and can be discharged during the night, according to economical aim.

In the second part of this section we will report the computational results corresponding to 2 working days in winter with the micro-hydro power plant in operation. The swing range of the battery is now set between 0 and $75 \%$. Three scenarios are shown: the first one with the energy storage system initially fully discharged is illustrated in Fig. 10, the second one with the ESS partially charged in shown in Fig. 11, and the last one with the battery initially fully charged is pointed out in Fig. 12.

The analysis of these three figures enables to make a comparison: we can observe that the more advantageous hours to discharge the battery are between 18:00 and 21:00, and the same result is obtained in all the cases. The difference is in the battery charging during the first day: in the first situation the energy storage system is completely charged in subsequent moments between 13:00 and 16:00; in the second case the ESS is initially partially charged (about $25 \%$ ) and the charging starts in a later moment approximately at 13:30; finally in the third case the battery is only discharged during the hours (from 18:00 to 21:00) with the highest electricity tariffs. There are not significant differences during the second working day because the initial condition with the ESS fully discharged is the same for all the three situations: the economical optimization follows the same logic of the previous day.

What is extremely visible in Figs. 10, 11 and 12 is the presence of high peaks in the curves of purchased energy. This point is determinated by the swing range between 0 and $75 \%$, which allows to buy a great quantity of energy. According to the economical aim, it is suitable buying energy to fully charge the battery, in order to discharge it when the electricity price is too expensive.

\section{A different energy storage strategy}

In this section a different resolution of the economical microgrid optimization problem is briefly outlined. The proposed approach maximizes two conflicting objectives: the economic gains and the energy storage life. As shown in Table 1, the lifetime of the battery storage depends on the swing range of a cycle of charge and discharge: the greater its use, the lower its lifetime.

Multiobjective optimization is a method to find the best solution between different, usually conflicting objectives.

\subsection{Multiobjective optimization}

Multiobjective optimization is an area of multiple criteria decision making, concerning with mathematical optimization problems involving more than one objective function to be optimized simultaneously. The two or more objective functions are at least partly conflicting and incommensurable, so there is the need to introduce the concept of Pareto optimality. For further details, see [4].

A solution is called Pareto optimal if none of the objective functions can be improved in value without impairment in some of the other objective values [4]. Without additional preference information, all Pareto optimal solutions can be considered 
R. De Leone et al.

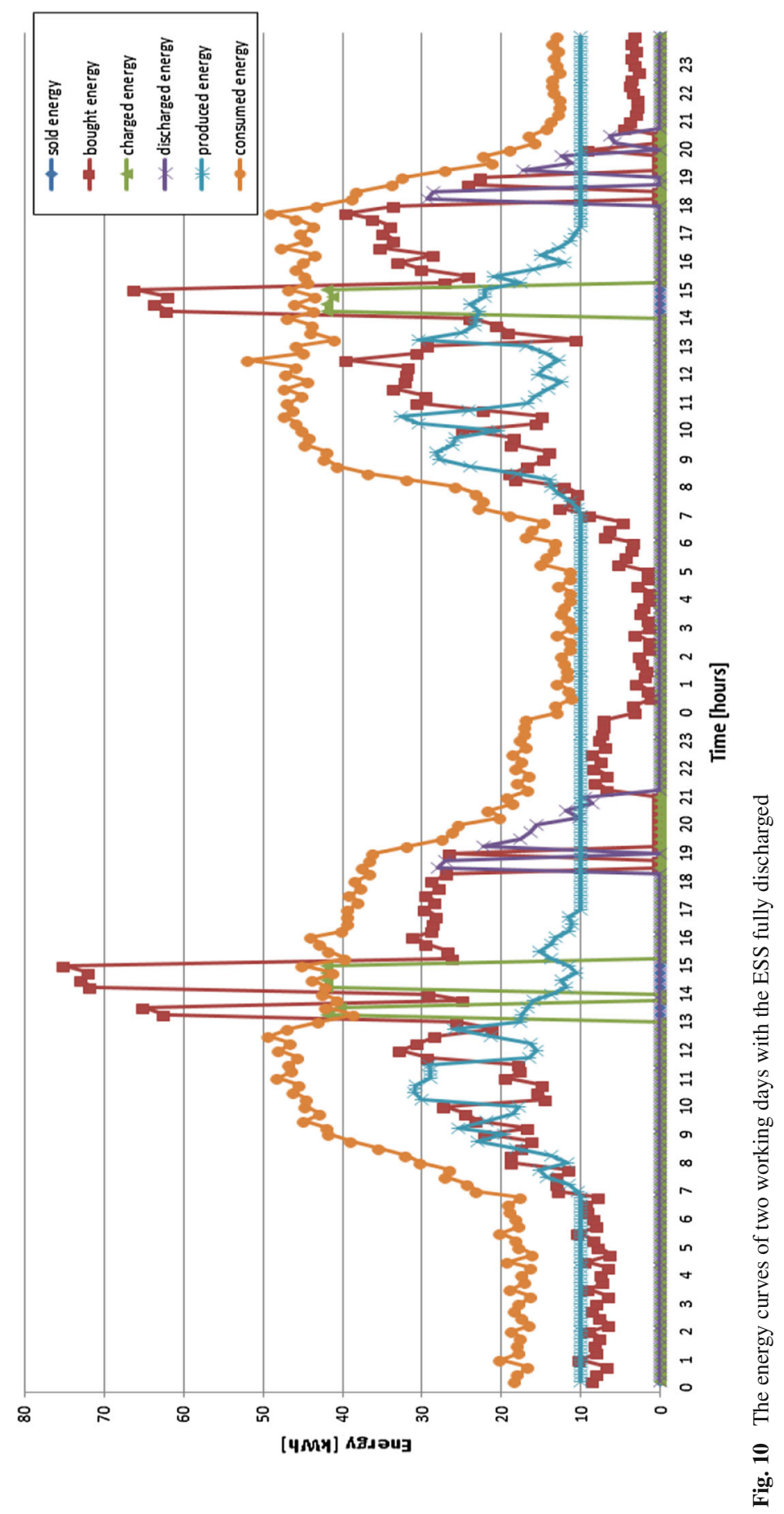




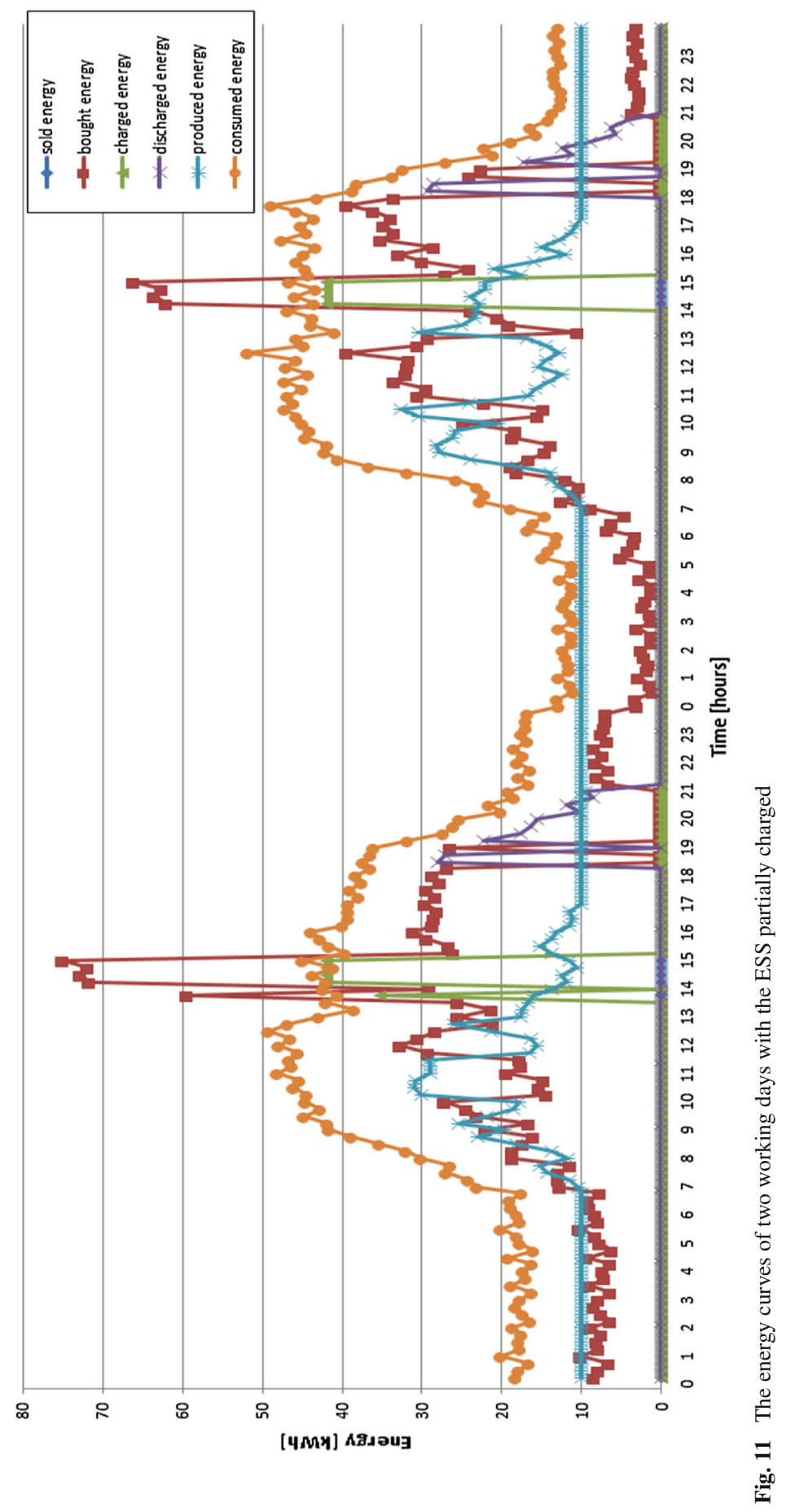


R. De Leone et al.

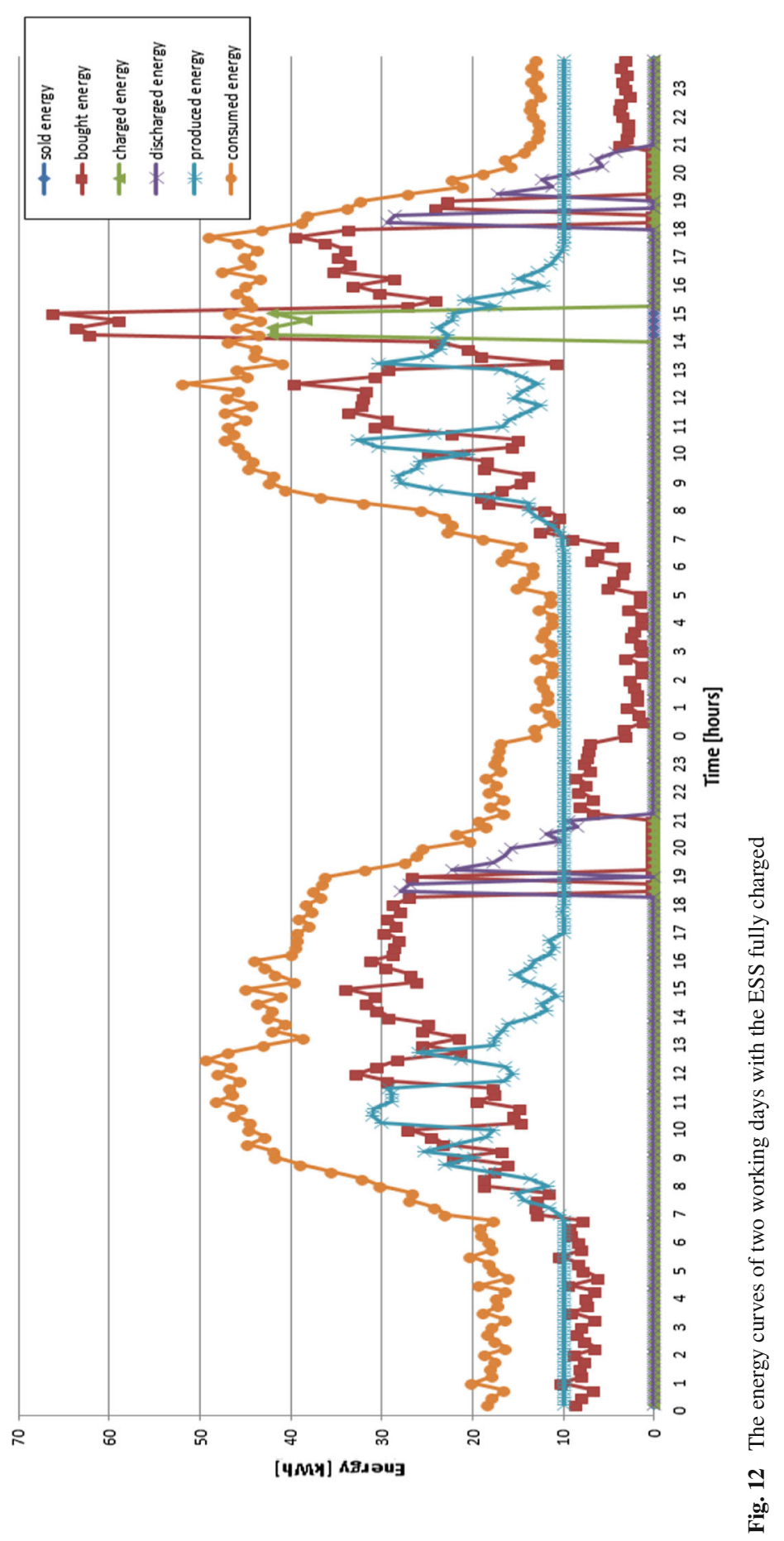




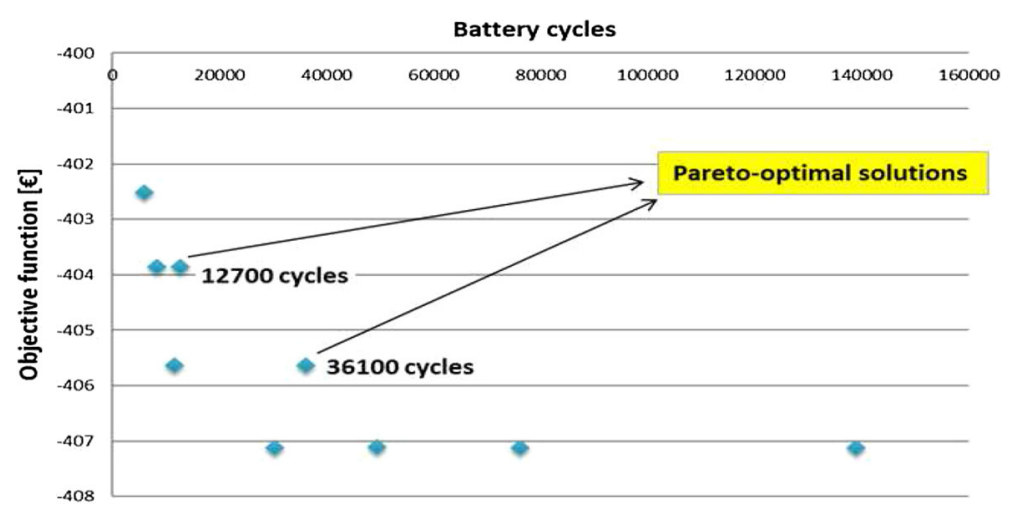

Fig. 13 The objective function values in relation to the lifetime battery cycles using different swing ranges of the storage

mathematically equally good and equally acceptable solutions of the multiobjective optimization problem. However, in practical problems it is generally desirable to obtain one point as a solution of the problem.

This is why - compared to single objective optimization - the element of the Decision Maker has to be added in multiobjective optimization. The decision maker is a person (or a group of people) who is supposed to have better insight into the problem and who can express preference relations between different solutions. The most preferred solution can be found using different philosophies: a posteriori method [4] is the one used to solve the multiobjective microgrid optimization problem.

\subsection{Problem definition}

In this situation, the economic optimization of the energy flows in the Leaf Community is not aimed at determining in advance how to manage the energy storage, but different problem scenarios are considered, each imposing constraints on the swing range of charging and discharging of the battery (see Eqs. 5, 6) and then deciding the interval which will be the best, based on the value of the objective function. All other constraints presented in Sect. 4.2 continue to hold. In the microgrid optimization problem the decision maker decides whether to give priority to lifetime of the battery or to economic gains, choosing between different Pareto optimal solutions.

\subsection{Computational results}

We report here one computational result corresponding to the previous two days in winter (a working day and a day off) with the micro-hydro power plant in operation and the energy storage at the minimum energy level with respect to the different scenarios of the storage.

In Fig. 13 on the horizontal axis there are the life cycles of the battery to maximize and on the ordinate axis there are the values of the objective function for each interval 
of charging and discharging of the energy storage, which are to maximize too. The two values highlighted are the Pareto optimal solutions in the microgrid optimization problem, the best satisfying the two objectives, not dominated by the other solutions. Clearly, the decision maker has to choose between the two Pareto optimal solutions, establishing which of the two objectives of the microgrid optimization cost function is preferable, in order to find the final optimal solution of the multiobjective optimization problem. If maximizing profits is the favourite aim, then the highest point has to be chosen, which penalizes the lifetime of the battery; otherwise, the preference is the lifetime of the energy storage.

\section{Conclusions}

A model to determine the optimum operation of a microgrid with respect to a load demand and with economical aim is constructed. The microgrid optimization problem includes a variety of energy sources that can be found in a microgrid: PV panels, microhydro power station and energy storage system. Constraints functions are added to the optimization problem to reflect some of the additional considerations on these devices. From the results obtained, it is clear that the optimization algorithm works very well taking into account all these constraints. Due to the time-varying nature of the consumptions and the variation of the battery state of charge, the system central controller need to respond to continuously changing operation conditions. Finally, two different energy storage strategies are illustrated: a-priori optimal strategy, in which the energy level in the storage is first established, and a-posteriori optimal strategy, where a decision maker has to choose between the Pareto optimal solutions of the multiobjective approach to the Microgrid Optimization Problem.

\section{Future work}

The actual contribution of the paper is to prove the effectiveness of the mixed integer linear programming into a real situation, such as Leaf Community microgrid. The focus is on the modeling part and on the solution of a real problem, not on the algorithmic one. Future work will envolved into decomposition of the different blocks, something similar to densing-Wolfe decomposition, which is beneficial for large problems to improve the algorithm efficiency. Stochastic inputs are well hoped in order to make the model more robust than now.

The introduction of new consumption buildings and renewable energy production plants will be inserted into the microgrid optimization problem.

Moreover, a study on a thermal energy storage (TES) will be led to introduce in the model the analysis of the thermal conditions of some buildings of the microgrid, with the aim of increasing the energy efficiency of the overall system thanks to load shifting.

Acknowledgments The authors express deep gratitude to the Loccioni Group, and in particular to Gino Romiti, innovator director, for providing dataset used in the present research study. The work of the first 
author is partly supported by a grant from GNCS-INdAM 2014 (Grant Metodi Numerici per l'ottimizzazione non lineare).

\section{References}

1. Ackermann, T., Andersson, G., Söder, L.: Distributed generation: a definition. Electric Power Syst. Res. 57(3), 195-204 (2001). doi:10.1016/S0378-7796(01)00101-8. http://www.sciencedirect.com/science/ article/pii/S0378779601001018

2. Basu, M.: Economic environmental dispatch of fixed head hydrothermal power systems using nondominated sorting genetic algorithm-ii. Appl. Soft Comput. 11(3), 3046-3055 (2011). doi:10.1016/j. asoc.2010.12.005

3. Chang, C., Wong, K., Fan, B.: Security-constrained multiobjective generation dispatch using bicriterion global optimisation. IEEE Proc. Gener. Transm. Distrib. 142, 406-414(8) (1995). http://digital-library. theiet.org/content/journals/10.1049/ip-gtd_19951806

4. Chankong, V., Haimes, Y.: Multiobjective Decision Making: Theory and Methodology. Dover Books on Engineering Series. Dover Publications, Incorporated (2008). http://books.google.it/books? id=9PQGGQAACAAJ

5. De Cosmis, S., De Leone, R.: Support vector machine for robust regression. In: COMTESSA (ed.) Proceedings of the CTW 2012 11th Cologne-twente workshop on graph and combinatorial optimization, pp. 96-99 (2012)

6. De Leone, R.: Support vector regression for time series analysis. In: Operations Research Proceedings 2010, pp. 33-38. Springer, Berlin (2011)

7. De Leone, R., Pietrini, M., Giovannelli, A.: Photovoltaic energy production forecast using support vector regression. Neural Comput. Appl. 26(8), 1955-1962 (2015). doi:10.1007/s00521-015-1842-y

8. Dhillon, J., Parti, S., Kothari, D.: Stochastic economic emission load dispatch. Electric Power Syst. Res. 26(3), 179 - 186 (1993). doi:10.1016/0378-7796(93)90011-3. http://www.sciencedirect.com/science/ article/pii/0378779693900113

9. Goodwin, P.: The holt-winters approach to exponential smoothing: 50 years old and going strong. Foresight Int. J. Appl. Forecast. 19, 30-33 (2010). http://EconPapers.repec.org/RePEc:for:ijafaa:y: 2010:i:19:p:30-33

10. Guan, X., Xu, Z., Jia, Q.S.: Energy-efficient buildings facilitated by microgrid. Smart Grid, IEEE Trans 1(3), 243-252 (2010). doi:10.1109/TSG.2010.2083705

11. Heslin, J.: A Multiobjective Production costing model for analyzing emissions dispatching and fuel switching. Case Western Reserve University (1990). http://books.google.it/books? id=mh07NwAACAAJ

12. Mohamed, F.A., Koivo, H.N.: Environmental/economic power dispatch of microgrid using multiobjective genetic algorithms. In: Proceedings of the International Renewable Energy Congress - IREC 2010, pp. 495-500. CMERP, Sousse, Tunisia (2010) (International Renewable Energy CongressIREC 2010, Sousse, Tunisia, November 5-7, 2010)

13. Mohamed, F.A., Koivo, H.N.: System modelling and online optimal management of microgrid with battery storage. In: International Conference on Renewable Energies and Power Quality (ICREPQ'07). EA4EPQ, Universidad de Sevilla, Sevilla (2007)

14. Morais, H., Kdr, P., Faria, P., Vale, Z.A., Khodr, H.: Optimal scheduling of a renewable microgrid in an isolated load area using mixed-integer linear programming. Renew. Energy 35(1), 151-156 (2010). doi:10.1016/j.renene.2009.02.031. http://www.sciencedirect.com/science/article/pii/ S0960148109001001

15. Narayanaswamy, B., Garg, V.K., Jayram, T.S.: Online optimization for the smart (micro) grid. In: Proceedings of the 3rd International Conference on Future Energy Systems: Where Energy, Computing and Communication Meet, e-Energy '12, pp. 19:1-19:10. ACM, New York (2012). doi:10.1145/ 2208828.2208847. http://doi.acm.org/10.1145/2208828.2208847

16. Parisio, A., Glielmo, L.: Energy efficient microgrid management using model predictive control. In: CDC-ECE, pp. 5449-5454. IEEE (2011). http://dblp.uni-trier.de/db/conf/cdc/cdc2011.html\# ParisioG11

17. Parisio, A., Glielmo, L.: A mixed integer linear formulation for microgrid economic scheduling. In: SmartGridComm, pp. 505-510. IEEE (2011). http://dblp.uni-trier.de/db/conf/smartgridcomm/ smartgridcomm2011.html\#ParisioG11 
18. Pepermans, G., Driesen, J., Haeseldonckx, D., Belmans, R., Dhaeseleer, W.: Distributed generation: definition, benefits and issues. Energy Policy 33(6), 787-798 (2005). doi:10.1016/j.enpol.2003.10. 004. http://www.sciencedirect.com/science/article/pii/S0301421503003069

19. Pesaran, B., Pesaran, M.H.: Time Series Econometrics Using Microfit 5.0: A User's Manual. Oxford University Press, Inc., New York (2010)

20. Vapnik, V.N.: The Nature of Statistical Learning Theory. Springer-Verlag New York Inc, New York (1995) 